\title{
Optimizing the timing of diagnostic testing after positive findings in lung cancer screening: a proof of concept radiomics study
}

Zixing Wang ${ }^{1}$, Ning $\mathrm{Li}^{1}$, Fuling Zheng ${ }^{2}$, Xin Sui ${ }^{2}$, Wei Han ${ }^{1}$, Fang Xue ${ }^{1}$, Xiaoli Xu ${ }^{2,3}$, Cuihong Yang ${ }^{1}$, Yaoda Hu ${ }^{1}$, Lei Wang ${ }^{1}$, Wei Song ${ }^{2^{*}}$ and Jingmei Jiang ${ }^{1 *}$ (D)

\begin{abstract}
Background: The timeliness of diagnostic testing after positive screening remains suboptimal because of limited evidence and methodology, leading to delayed diagnosis of lung cancer and over-examination. We propose a radiomics approach to assist with planning of the diagnostic testing interval in lung cancer screening.

Methods: From an institute-based lung cancer screening cohort, we retrospectively selected 92 patients with pulmonary nodules with diameters $\geq 3 \mathrm{~mm}$ at baseline (61 confirmed as lung cancer by histopathology; 31 confirmed cancer-free). Four groups of region-of-interest-based radiomic features $(n=310)$ were extracted for quantitative characterization of the nodules, and eight features were proven to be predictive of cancer diagnosis, noise-robust, phenotype-related, and non-redundant. A radiomics biomarker was then built with the random survival forest method. The patients with nodules were divided into low-, middle- and high-risk subgroups by two biomarker cutoffs that optimized time-dependent sensitivity and specificity for decisions about diagnostic workup within 3 months and about repeat screening after 12 months, respectively. A radiomics-based follow-up schedule was then proposed. Its performance was visually assessed with a time-to-diagnosis plot and benchmarked against lung RADS and four other guideline protocols.
\end{abstract}

Results: The radiomics biomarker had a high time-dependent area under the curve value $(95 \% \mathrm{Cl})$ for predicting lung cancer diagnosis within 12 months; training: 0.928 (0.844, 0.972), test: $0.888(0.766,0.975)$; the performance was robust in extensive cross-validations. The time-to-diagnosis distributions differed significantly between the three patient subgroups, $p<0.001: 96.2 \%$ of high-risk patients $(n=26)$ were diagnosed within 10 months after baseline screen, whereas $95.8 \%$ of low-risk patients $(n=24)$ remained cancer-free by the end of the study. Compared with the five existing protocols, the proposed follow-up schedule performed best at securing timely lung cancer diagnosis (delayed diagnosis rate: $<5 \%$ ) and at sparing patients with cancer-free nodules from unnecessary repeat screenings and examinations (false recommendation rate: $0 \%$ ).

\footnotetext{
*Correspondence: cjr.songwei@vip.163.com;

jingmeijiang@ibms.pumc.edu.cn

${ }^{1}$ Department of Epidemiology and Biostatistics, Institute of Basic

Medical Sciences, Chinese Academy of Medical Sciences/School of Basic

Medicine, Peking Union Medical College, Beijing, China

${ }^{2}$ Department of Radiology, Peking Union Medical College Hospital,

Chinese Academy of Medical Sciences, Beijing, China

Full list of author information is available at the end of the article
}

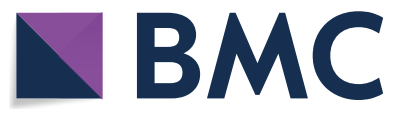

(c) The Author(s) 2021. This article is licensed under a Creative Commons Attribution 4.0 International License, which permits use, sharing, adaptation, distribution and reproduction in any medium or format, as long as you give appropriate credit to the original author(s) and the source, provide a link to the Creative Commons licence, and indicate if changes were made. The images or other third party material in this article are included in the article's Creative Commons licence, unless indicated otherwise in a credit line to the material. If material is not included in the article's Creative Commons licence and your intended use is not permitted by statutory regulation or exceeds the permitted use, you will need to obtain permission directly from the copyright holder. To view a copy of this licence, visit http://creativeco mmons.org/licenses/by/4.0/. The Creative Commons Public Domain Dedication waiver (http://creativecommons.org/publicdomain/ zero/1.0/) applies to the data made available in this article, unless otherwise stated in a credit line to the data. 
Conclusions: Timely management of screening-detected pulmonary nodules can be substantially improved with a radiomics approach. This proof-of-concept study's results should be further validated in large programs.

Keywords: Lung cancer screening, Radiomics biomarker, Follow-up, Pulmonary nodule management, Timedependent analysis

\section{Background}

Low-dose CT screening has been widely accepted as a means of mortality reduction and early detection of lung cancer [1-3]. It is a long-term rather than one-take effort because large numbers of indeterminate pulmonary nodules may require diagnostic workups, follow-up scans, or annual repeat screenings $[4,5]$. Currently, recommendations on the time targets for follow-up are based on nodules' diameter and solidity [6, 7]. However, it has been shown that these features are insufficient to measure nodules' complex appearance [8], and visual interpretations of solidity are prone to inter-rater variability $[9,10]$. The timeliness of follow-up after positive screening remains suboptimal because of limited evidence [11]. Innovating the way we help patients with nodules to make subsequent decisions is important, as we must weigh the benefits of early cancer diagnosis against the danger and cost of over-investigating unaggressive nodules.

In this study, we present a radiomics pipeline to select, synthetize, and recode radiomics data extracted from CT images and produce follow-up schedules to facilitate timely management of screening-detected nodules. We show the potential clinical impact of this approach by comparing its performance against that of five existing protocols specified in current guidelines.

\section{Methods}

\section{Study participants}

The study's subjects were from an institute-based lung cancer screening cohort. The participants were those who underwent low-dose CT screening in August 2014-August 2018 and had at least one noncalcified pulmonary nodule detected. The inclusion criteria for a baseline screening were age $40-80$ years and nodule diameter $\geq 3 \mathrm{~mm}$ (defined as the mean of the major and minor axis lengths, rounded to the nearest integer). The exclusion criteria were: (1) pregnancy; (2) severe illness of the brain, heart, or kidney; (3) other conditions not suitable for $\mathrm{CT}$ examination, determined by radiologists; (4) already hospitalized or transferred from hospitals for further workup; (5) distant residence that prevented timely follow-up.

By the end of May 2019, we had included 61 cases diagnosed with lung cancer (including 52 with histopathologically confirmed adenocarcinoma, 7 with adenocarcinoma in situ, 1 with squamous cell carcinoma, and 1 with metastatic carcinoma of the prostate), and we retrospectively selected 31 cancer-free patients with nodules who met any of the following conditions: (1) histopathologically confirmed benign lesion by pathology test ( $\mathrm{n}=24$, including 17 with hamartoma, 3 with pneumocytoma, 3 with inflammation, and 1 with carcinoid); (2) nodule disappeared or decreased in size in follow-up screening $(\mathrm{n}=3)$; (3) no sign of malignancy during follow-up for at least 2 years $(n=4)$. Cancer-free status was cross-validated through medical records to minimize the effects of missed detection by histopathology tests.

\section{Data collection}

We used a site-based research database to collect, store, and perform quality control of the following data: (1) demographic information, including age at baseline, sex, personal and family (first-degree) cancer history, and smoking status (current smoking defined as $\geq 10$ packyears; quit smoking defined as $\geq 5$ years' cessation); (2) outcome of follow-up, including date of lung cancer diagnosis (analyzed as time/status outcome), specific pathological type, and cancer stage at diagnosis; (3) semantic phenotypes of the nodules, recorded as categorical variables, including nodule type (solid, part-solid, or nonsolid), lobular, specular, juxtapleural, and pleura tag.

Baseline and follow-up CT images were acquired according to standardized protocols using a SOMATOM Definition Flash scanner, a SOMATOM Force scanner, and others. Images were reconstructed up to a thickness of $5.0 \mathrm{~mm}$ with a spacing of no more than $1.5 \mathrm{~mm}$, stored as DICOM files, and retrieved from our Picture Archiving and Communication Systems.

\section{Radiomics data generation}

For each patient with nodules, one baseline CT image with the maximum nodule area in the transaxial plane was selected for primary analysis. The same rule was applied if there was more than one pulmonary nodule. Temporal changes in radiomic features were analyzed among patients with nodules who had one or more repeat $\mathrm{CT}$ scans during follow-up.

Regions-of-interest were delineated following the multi-step interactive process detailed in Additional file 1: Method S1. Four groups of region-of-interestbased radiomic features, which have been extensively used in radiomics studies [12, 13], were extracted for 
quantitative characterization of the nodules: 21 shape features (Euclidean and fractal), 8 intensity features (histogram-based statistics), 41 texture features (gray-level co-occurrence matrix and run-length matrix), and 240 wavelet features (Additional file 1: Method S2).

\section{Biomarker development}

The proposed follow-up schedules were based on a composite radiomic biomarker developed using the random survival forest (RSF) method [14] that discriminates between the time-to-diagnosis distributions of patient subgroups. To increase interpretability and avoid over-fitting, only a few predictive, noise-robust, clinically meaningful, non-redundant radiomic features were selected as inputs to the RSF (see technical details about feature selection and biomarker development in Additional file 1: Method S3-4). This feature selection process was performed in a training set that was composed of $67 \%$ the participants; A radiomics biomarker was then trained in this training set and tested in the rest of the participants. A cross-validation approach (by approximately equal sized and mutually exclusive folds; no stratification variable applied) was used to evaluate the robustness of the results. The biomarker's performance was also examined after being combined with demographic and semantic phenotype variables to investigate whether the addition of such information is necessary.

\section{Schedule design}

The radiomics biomarker was used to stratify the patients with nodules in a way that resembles previously published nodule management protocols [6, 7, 15-17]. To minimize delayed cancer diagnosis, a "low" cutoff value was selected to provide high time-dependent sensitivity to the decision about early diagnostic workup (within 3 months). Similarly, to reduce unnecessary follow-up, a "high" cutoff was selected to provide high time-dependent specificity to the decision about repeat screening (after 12 months). Nodule management plans were then made for the low-, middle-, and high-risk patient subgroups, defined as having biomarker values below, between, and above the cutoffs, respectively. The proposed schedules' performance was visually assessed with a time-to-diagnosis plot and benchmarked against the protocols recommended in five expert consensus-based guidelines in a contingency table.

\section{Statistical analysis}

Because our research goal concerns the timing of diagnosis and follow-up rather than binary classification, more than one time point of interest (e.g., 3 months, 1 year) was selected to reclassify each study participant as a "cumulative case" (diagnosed with lung cancer before the time of interest) or "dynamic control" (not diagnosed with cancer by the time of interest, including lung cancers diagnosed later and patients with cancerfree nodules). This time-dependent definition was based on Heagerty's analysis framework [18] and allows us to evaluate the performance of potential nodule descriptors and the composite biomarker at predicting lung cancer diagnosis within several time intervals. A time-dependent version of the area under the curve metric (termed AUCt) was then calculated [18]. The bootstrap method (resampling 200 times) was used to estimate 95\% confidence intervals (CIs) where indicated.

In the calculation of sample size, a ratio ranging from 1:2 to 2:1 was applied to allow the numbers of "cumulative cases" and "dynamic controls" to vary according to different time points of interest. For an expected AUCt that ranges from 0.7 to 0.9 , we need 6 to 60 cases and 60 to 6 controls at different time points to achieve a power of 0.9 at a significance level of 0.050 (two-sided). With the available sample, the statistical powers of a significance test for AUCt values of 0.7 or above at 3 months, 6 months, and 12 months are $\geq 0.90,0.92$, and 0.93, respectively.

All statistical tests were two-sided, with a significance level of $p=0.050$, and were performed with $\mathrm{R}$ version 3.5.2.

\section{Results}

\section{Description of study participants}

Most of the 61 patients with lung cancer were diagnosed at an early stage $(7,50$, and 1 patient at stage 0, I, and II, respectively). Three patients were diagnosed at stage III or IV. As shown in Table 1, they had no significant differences from the cancer-free group in terms of age, sex, smoking status, or personal and family cancer history; all $p>0.050$. However, the cancer group had a significantly higher frequency of follow-up screens over the cancerfree group; $p=0.033$. Regarding characteristics of the nodules, there were no significant differences between the two groups in terms of diameter or semantic phenotypes including lobulation, speculation, juxtapleural, and pleura tag (all $p>0.050$ ). Nodules in both groups were dominated by a part-solid type, but there was a higher proportion of solid nodules in the cancer-free group $(p<0.001)$. Further, most malignant nodules were in the upper lobes of the lung, whereas nearly half of the nodules in the cancer-free group were in the lower lobes $(p=0.026)$.

\section{Radiomic features selected}

Eight radiomic features were selected following the flowchart depicted in Fig. 1. They included a shape feature (circularity), three intensity features (variance, kurtosis, 
Table 1 Characteristics of Study Participants and Pulmonary Nodules at Baseline

\begin{tabular}{|c|c|c|c|}
\hline Characteristics & Lung cancer $(n=61)$ & Cancer-free $(n=31)$ & $p$-value \\
\hline Age (year), mean $\pm S D$ & $58.1 \pm 10.1$ & $55.7 \pm 10.8$ & 0.276 \\
\hline \multicolumn{4}{|l|}{ Sex, n (\%) } \\
\hline Man & $15(24.6)$ & $12(38.7)$ & \multirow[t]{2}{*}{0.226} \\
\hline Woman & $46(75.4)$ & $19(61.3)$ & \\
\hline \multicolumn{4}{|l|}{ Smoking status, n (\%) } \\
\hline Currently smoke & $3(4.9)$ & $2(6.5)$ & \multirow[t]{3}{*}{0.847} \\
\hline Quit smoke & $2(3.3)$ & $0(0.0)$ & \\
\hline Never smoke & $56(91.8)$ & 29 (93.6) & \\
\hline \multicolumn{4}{|l|}{ Cancer history, n (\%) } \\
\hline Personal & $8(13.1)$ & $1(3.2)$ & 0.264 \\
\hline Family & $1(1.6)$ & $0(0.0)$ & $>.999$ \\
\hline \multicolumn{4}{|l|}{ No. of screening, n (\%) } \\
\hline 1 & $33(54.1)$ & $25(80.7)$ & \multirow[t]{3}{*}{0.033} \\
\hline 2 & $22(36.1)$ & $4(12.9)$ & \\
\hline$\geq 3$ & $6(9.8)$ & $2(6.5)$ & \\
\hline \multicolumn{4}{|l|}{ Nodule location, n (\%) } \\
\hline Left upper lobe & $19(31.2)$ & $8(25.8)$ & \multirow[t]{5}{*}{0.024} \\
\hline Left lower lobe & $6(9.8)$ & $10(32.3)$ & \\
\hline Right upper lobe & $29(47.5)$ & $7(22.6)$ & \\
\hline Right middle lobe & $1(1.6)$ & $1(3.2)$ & \\
\hline Right lower lobe & $6(9.8)$ & $5(16.1)$ & \\
\hline \multicolumn{4}{|l|}{ Diameter (mm) } \\
\hline$<6$ & $2(3.3)$ & $0(0.0)$ & \multirow[t]{6}{*}{0.807} \\
\hline $6 \sim$ & $3(4.9)$ & $1(3.2)$ & \\
\hline $8 \sim$ & $8(13.1)$ & $7(22.6)$ & \\
\hline $10 \sim$ & $27(44.3)$ & $12(38.7)$ & \\
\hline $15 \sim$ & $12(19.7)$ & $5(16.1)$ & \\
\hline $20 \sim$ & $9(14.8)$ & $6(19.4)$ & \\
\hline \multicolumn{4}{|l|}{ Nodule type } \\
\hline Solid & $4(6.6)$ & $11(35.5)$ & \multirow[t]{3}{*}{$<0.001$} \\
\hline Part-solid & $42(68.9)$ & $18(58.1)$ & \\
\hline Non-solid & $15(24.6)$ & $2(6.5)$ & \\
\hline \multicolumn{4}{|c|}{ Other semantic phenotype } \\
\hline Lobular & $35(57.4)$ & $20(64.5)$ & 0.653 \\
\hline Spiculated & $20(32.8)$ & $7(22.6)$ & 0.344 \\
\hline Juxtapleural & $9(14.8)$ & $10(32.3)$ & 0.061 \\
\hline Pleural tag & $10(16.4)$ & $2(6.5)$ & 0.326 \\
\hline
\end{tabular}

In cases in which more than one pulmonary nodule was present, data on nodule characteristics are shown for the one that had the greatest area in the transaxial plane

${ }^{\text {a }}$ Current smoker defined as $\geq 10$ pack-years; quit smoking defined as $\geq 5$ years' cessation

energy), three texture features (cluster shade, maximum probability, long-run high gray-level emphasis mean [LongHEM]), and a wavelet feature (long-run emphasis mean on approximation signal). All selected features had high predictive accuracy (AUCt $\geq 0.7$ at $\mathrm{t}=12$ months) and were robust to image noise (intraclass correlation coefficient $>0.9$ ), non-redundant (variance inflation factor $<7$ in collinearity diagnostics), and significantly related to at least one of the five semantic phenotypes. Additional file 1: Table S1 provides more information about the eight selected radiomic features' data distributions and other characteristics.

Figure 2 illustrates the potential usefulness of LongHEM as an example of the selected features. Solid, part-solid, and non-solid nodules had markedly different LongHEM values (Fig. 2a), $p<0.001$. This radiomic 


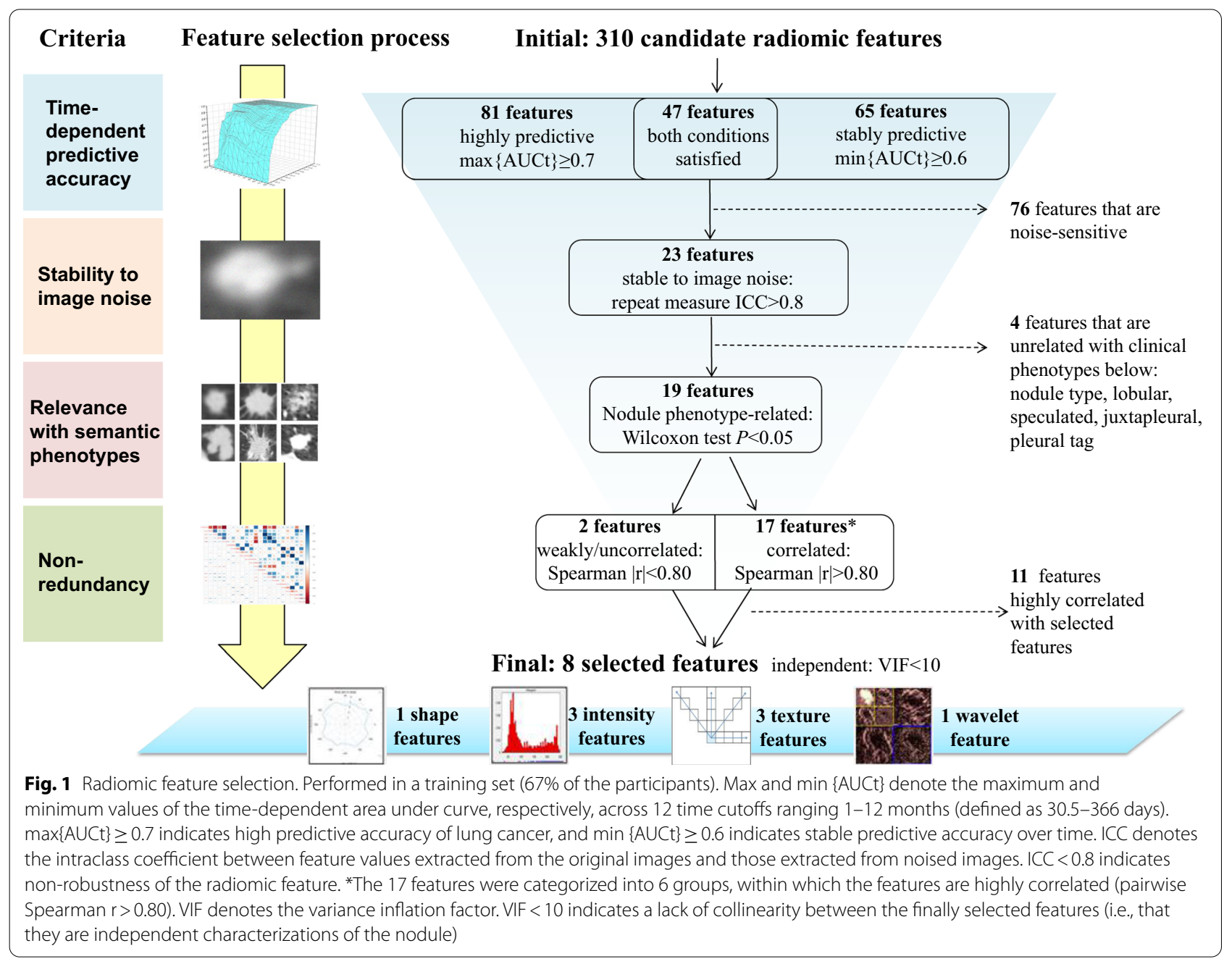

feature performed well at differentiating patients with cancer from cancer-free patients, especially when combined with the energy feature (Fig. 2b). Compared with nodule diameter, the change in LongHEM was more sensitive to the time interval between baseline and repeat screenings in patients with cancer (Fig. 2c). The sensitivity to temporal change was further validated by examining the CT images of a nodule detected in the upper left lung of a male patient aged 74 years at detection, who underwent two repeat screenings after 630 and 768 days and was diagnosed with lung cancer at age 77 years. The relative change in LongHEM was more marked and occurred earlier than that of the nodule's diameter (Fig. 2d).

\section{Predictive performance of the radiomics biomarker}

Figure 3 presents the radiomics biomarker's timedependent performance. For prediction of lung cancer diagnosis within 3,6 , and 12 months, respectively, the biomarker had AUCt values of 0.837, 0.887, and 0.928 on the training dataset and $0.740,0.852$, and 0.888 on the test dataset; all $p<0.050$. The biomarker performed much better than nodule diameter; the latter showed AUCt values of $0.616,0.578,0.569$ on the training dataset and $0.673,0.641,0.683$ on the test dataset for prediction of lung cancer diagnosis within 3,6 , and 12 months, respectively; all $p>0.050$.

The biomarker's performance was robust in extensive cross-validations (Additional file 1: Table S2). For instance, the median (interquartile range) of AUCt at 12 months was $0.870(0.750,0.919)$ in a tenfold cross validation, indicating a small chance of over-fitting. No improvement was observed, for example, by further adding the semantic phenotypes ( $\mathrm{AUCt}=0.879$ at 12 months), nodule location (0.859), demographic information (0.866), or a combination of these nonradiomic variables (0.857), irrespective of the request for additional input from radiologists and participants. 

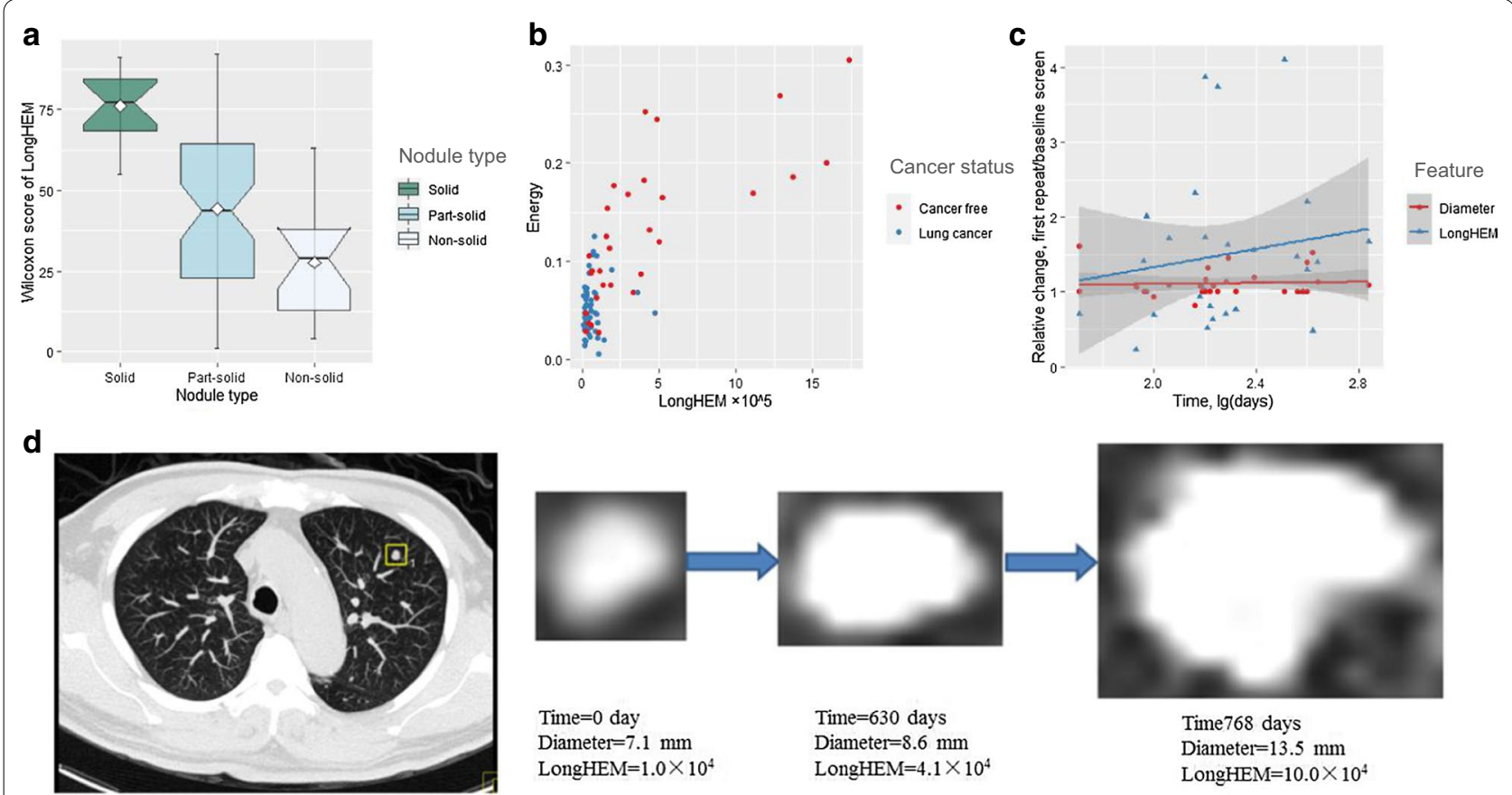

Time768 days

Diameter $=13.5 \mathrm{~mm}$

LongHEM=10.0 $\times 10^{4}$

Fig. 2 Potential value of a radiomic feature for interpreting CT images in lung cancer screening. LongHEM: long-run high gray-level emphasis mean. In (a), the distributions of LongHEM was compared between nodules with different types. In (b), the status of the nodules was classified by the end of the study. In (c), the regression slope is 0.610 vs. 0.034 increase per log[day] for the relative change in LongHEM vs. diameter. One influential data point $(1.71,21.52)$ for the temporal change in LongHEM is not shown, which corresponds to a relative change of 21.52 in LongHEM in 51 days from baseline to the first repeat screen in a patient finally diagnosed with lung adenocarcinoma. In (d), the temporal changes of LongHEM and diameter of a malignant nodule were compared
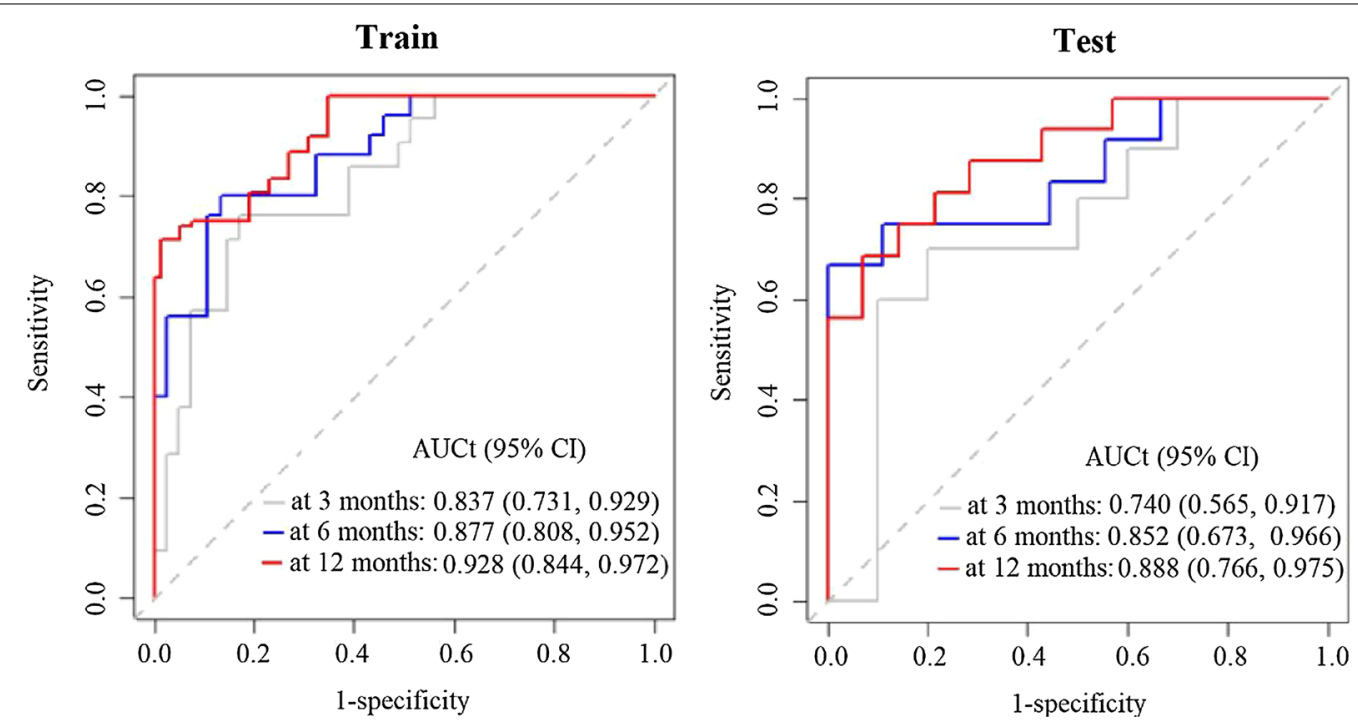

Fig. 3 Time-dependent performance of a radiomics biomarker in training and test datasets. The training and test datasets had 62 and the 30 observations, respectively. AUCt $(95 \% \mathrm{Cl})$, time-dependent area under the curve (95\% confidence interval)

\section{Clinical utility of the proposed nodule management schedules}

The time to diagnosis of lung cancer differed significantly between the three patient subgroups $(p<0.001$; Fig. 4$)$. All but 1 of the 26 patients $(96.1 \%)$ with nodules classified as high-risk were diagnosed within 10 months 


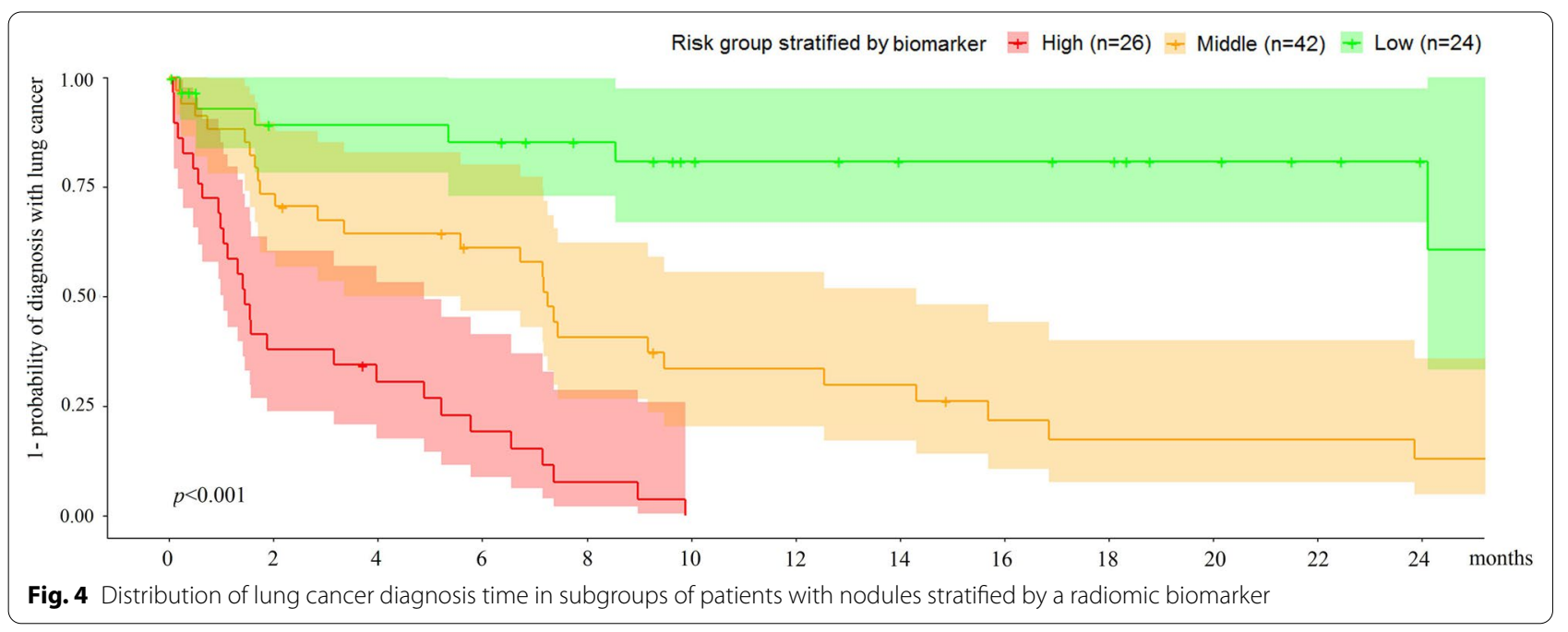

after the baseline screening. Similarly, all but 1 of the 24 patients $(95.8 \%)$ with nodules classified as low-risk remained cancer-free by the end of the study. When 30 and 75 were used as the biomarker cutoff values to stratify the patients, the time-dependent sensitivity (95\% CI) was $0.968(0.896,1.000)$ at 3 months, and the specificity $(95 \% \mathrm{CI})$ was $0.975(0.919,1.000)$ at 12 months.

\section{Benchmark against existing protocols}

We benchmarked the radiomics-based follow-up schedule against those recommended by five expert consensus-based guideline protocols (Table 2). If the AATS Guideline protocol [17] had been followed, delayed diagnosis would have occurred in over $90 \%$ of patients with lung cancer who were actually diagnosed within 3 months, as that protocol tended to be conservative and recommended follow-ups "in 3-6 months" or "in 6 months" more frequently than an immediate diagnostic workup or earlier follow-up. If the ACCP [16] or China Guideline [7] protocols had been used, there would have been a substantial number of unnecessary early followup screens (i.e., nearly $90 \%$ of the cancer-free patients would have been recommended for a follow-up "within 3 months"). The Lung-RADS [15] or NCCN Guideline [6] protocols frequently recommend diagnostic workup such as contrast CT and/or PET/CT even for nearly $60 \%$ of the cancer-free patients; in addition, cancer diagnosis might have been delayed by the recommendation of "annual screening" in approximately 25\% of patients with lung cancer who were actually diagnosed within 3 months or 12 months. On the basis of the proposed radiomics approach, fewer than $5 \%$ of the patients with lung cancer would have their diagnoses delayed because of the recommendation of annual follow-up, and $0 \%$ of the cancer-free patients with nodules would have unnecessarily undergone a diagnostic workup.

\section{Discussion}

In this study, we developed a radiomics biomarker on the basis of eight predictive, noise-robust, non-redundant radiomic features. The clinical usefulness of those features as nodule descriptors was justified by their high relevance to semantic phenotypes, higher discriminative value, and greater temporal sensitivity than nodule diameter. The biomarker had high time-dependent predictive accuracy for lung cancer and could well differentiate subgroups of patients with nodules according to their distinct times to cancer diagnosis. When benchmarked against five current guideline protocols, the proposed approach performed best at reducing both delayed and over-diagnosis rates, suggesting the great potential of applying radiomics to secure a timely cancer diagnosis as well as sparing patients with unaggressive nodules from unnecessary diagnostic testing in lung cancer screening.

Automatic detection of pulmonary nodules and prediction of their malignancy and benignity have been extensively investigated [19-21]. The major differences of our study from these works are that we applied radiomics to schedule the timeliness of nodule management and used a new analysis method to allow for the addition of the time dimension. There are some advantages associated with this change. First, some cancers can be diagnosed immediately but some cannot (e.g., as long as 33.5 months in this study). Compared with treating them as one group for prediction, it is more clinically meaningful to determine whether the patient can wait a while (e.g., 6 months, 12 months) to make a judgment through follow-up. The time-dependent definitions of case and control are more pertinent to the longitudinal nature of 
Table 2 Benchmark of Proposed Nodule Management Schedule against Existing Protocols

\begin{tabular}{|c|c|c|c|c|c|}
\hline \multirow[t]{2}{*}{ Recommendations } & \multirow{2}{*}{$\begin{array}{l}\text { No. of participants (\%, } \\
\text { by column) }\end{array}$} & \multicolumn{3}{|c|}{ Lung Cancer diagnosed } & \multirow{2}{*}{$\begin{array}{l}\text { Cancer-free } \\
\text { by study end }(n=31)\end{array}$} \\
\hline & & $\begin{array}{l}\text { within } 3 \text { mos } \\
(n=31)\end{array}$ & $\begin{array}{l}\text { within } 3-12 \text { mos } \\
(n=20)\end{array}$ & $\begin{array}{l}\text { after } 12 \text { mos } \\
(n=10)\end{array}$ & \\
\hline \multicolumn{6}{|l|}{ AATS guideline, 2012} \\
\hline Diagnostic workup & $13(14.1)$ & $3(9.7)$ & $0(0.0)$ & $1(10.0)$ & $9(29.0)$ \\
\hline Follow-up in 3 mos & $2(2.2)$ & $0(0.0)$ & $0(0.0)$ & $0(0.0)$ & $2(6.5)$ \\
\hline Follow-up in 3-6 mos & $52(56.5)$ & $21(67.7)$ & $15(75.0)$ & $4(40.0)$ & $12(38.7)$ \\
\hline Follow-up in 6 mos & $25(27.2)$ & $7(22.6)$ & $5(25.0)$ & $5(50.0)$ & $8(25.8)$ \\
\hline \multicolumn{6}{|l|}{ ACCP Guideline, 2013} \\
\hline Diagnostic workup & $1(1.1)$ & $0(0.0)$ & $0(0.0)$ & $0(0.0)$ & $1(3.2)$ \\
\hline Follow-up at 3 mos & $72(78.2)$ & $23(74.2)$ & $15(75.0)$ & $8(80.0)$ & $26(83.9)$ \\
\hline Follow-up in 6-12 mos & $2(2.2)$ & $0(0.0)$ & $0(0.0)$ & $0(0.0)$ & $2(6.5)$ \\
\hline Annual screen & $16(17.4)$ & $8(25.8)$ & $5(25.0)$ & $1(10.0)$ & $2(6.5)$ \\
\hline No further evaluation & $1(1.1)$ & $0(0.0)$ & $0(0.0)$ & $1(10.0)$ & $0(0.0)$ \\
\hline \multicolumn{6}{|l|}{ China Guideline, 2018} \\
\hline Follow-up after 1 mos $^{a}$ & $32(34.8)$ & $15(48.4)$ & $6(30.0)$ & $0(0.0)$ & $11(35.5)$ \\
\hline Follow-up after 3 mos & $56(60.9)$ & $15(48.4)$ & $13(65.0)$ & $9(90.0)$ & $19(61.3)$ \\
\hline Annual screen & $4(4.3)$ & $1(3.2)$ & $1(5.0)$ & $1(10.0)$ & $1(3.2)$ \\
\hline \multicolumn{6}{|c|}{ Lung-RADS, 2019 or NCCN Guideline, 2020 b } \\
\hline Diagnostic workup & $42(45.7)$ & $16(51.6)$ & $7(35.0)$ & $1(10.0)$ & $18(58.1)$ \\
\hline Follow-up in 3 mos & $14(15.2)$ & $0(0.0)$ & $3(15.0)$ & $2(20.0)$ & $9(29.0)$ \\
\hline Follow-up in 6 mos & $18(19.6)$ & $7(22.6)$ & $5(25.0)$ & $4(40.0)$ & $2(6.5)$ \\
\hline Annual screen & 18 (19.6) & $8(25.8)$ & $5(25.0)$ & $3(30.0)$ & $2(6.5)$ \\
\hline \multicolumn{6}{|c|}{ Proposed radiomics approach } \\
\hline Diagnostic workup & $26(28.3)$ & $19(61.3)$ & $6(30.0)$ & $1(10.0)$ & $0(0.0)$ \\
\hline Follow-up in 3 mos & $42(45.7)$ & $11(35.5)$ & $14(70.0)$ & $9(90.0)$ & $8(25.8)$ \\
\hline Annual screen & $24(26.1)$ & $1(3.2)$ & $0(0.0)$ & $0(0.0)$ & $23(74.2)$ \\
\hline
\end{tabular}

AATS: American Association for Thoracic Surgery; ACCP: American College of Chest Physicians; Lung-RADS: Lung CT Screening Reporting \& Data System; NCCN: National Comprehensive Cancer Network

a After tentative anti-inflammatory therapy

${ }^{b}$ Lung-RADS (baseline screening) and NCCN guidelines have only small differences regarding management of perifissural nodules and the diameter criteria for different follow-up timings among non-solid nodules, which did not result in a difference in terms of nodule management with our data

lung cancer screening. Second, challenges with defining a disease-free group using a "gold standard" arise in the screening setting because few cancer-free participants undergo histopathology tests [5]. Their time to lung cancer diagnosis is censored, as it should be viewed from a lifetime horizon. The time-dependent analysis can properly employ this censored information, whereas simply ignoring this idea or treating the cancer-free group as non-diseased would result in bias. Third, by incorporating the temporal information, the proposed method can contribute to more precise risk assessment of lung cancer. The method can also address screening-related issues such as the harms associated with over-diagnosis (e.g., repeat exposure to radiation, invasive diagnostic procedures) and delayed diagnosis and intervention [2], all of which are core to the interests of screening participants. According to a recent review from the Population-based Research to Optimize the Screening Process Consortium
[11], timely follow-up for positive cancer screening results remains suboptimal because of the low quality of available evidence across cancers. The proposed approach could outline an important step in addressing these challenging issues.

One of the major concerns with radiomics is whether radiomic features are as reliable as has been reported $[22,23]$. In view of this, we adopted very stringent feature selection criteria. Among the reasons for exclusion listed in the flowchart, non-robustness to image noise was a particular consideration in our study beyond the level that was applied in other studies [18, 24, 25]. Noisesensitivity was important in our study because it is a unique issue in low-dose $\mathrm{CT}$ and could affect the stability of the results if different modality parameters or reconstruction algorithms are adopted [23]. We found that the majority of sophisticated radiomic features, such as wavelet-based features, are very sensitive to image noise 
and less relevant to semantic phenotypes, despite the fact that some have high predictive value. This finding indicates that there may be a balance between complexity, interpretability, and suitability in the search for new nodule descriptors. For this reason, we did not resort to $3 \mathrm{D}$ features, given that the results with $2 \mathrm{D}$ features were satisfactory and saved computing time for easier clinical uptake. Further, in lieu of semantic phenotypes, which are subject to moderate-high inter-rater variation $[9,10]$, the selected radiomic features could provide automatic (and thus more reliable) quantification of nodule characteristics. Clinical confidence in the use of these radiomic features may be improved by considering the following: first, as shown by our results, they were naturally associated with the semantic phenotypes commonly used by radiologists. Second, the addition of semantic phenotype variables did not improve predictive performance, meaning that the radiomic features already carry such qualitative information and thus may substantially reduce human labor. Third, the selected radiomics features' clinical value has been suggested in other studies. For instance, kurtosis and energy, as measures of the "tailedness" and homogeneity of the intensity distribution, showed high variable importance in our model (Additional file 1: Figure S1) and have been reported to be useful for discrimination between benign and malignant nodules [24, 26], helpful for prediction of prognosis, and associated with gene expression in lung cancer [27].

Among existing protocols, Lung-RADS has been widely accepted as a reliable tool, and its performance is especially accurate when previous images are available [28]. However, the performance of Lung-RADS has been shown to deteriorate on the baseline screening, when no priors are available [29]. The proposed radiomics approach performed much better than Lung-RADS and other protocols at decision making following the baseline screen. However, the low frequency of repeated screens prevented us from planning subsequent decisions. After this proof-of-concept study, we plan to apply the proposed time-dependent analysis framework to serial image data (recently termed delta-radiomics [30]) with a large sample size. This will hopefully contribute to refining dynamic management.

This study is limited in several aspects. First, its external validity is limited by the narrow spectrum of diseases investigated (particularly, most of the cancers were adenocarcinoma, a finding similar to other reports from China [20]). Second, the observed temporal data could have been affected by delayed or over-diagnosis. Third, the extraction of radiomic features is intrinsically repeatable, but variability may be introduced by the semiautomatic segmentation method. Fourth, the cancer-free group received significantly fewer follow-up screenings than the cancer group, and thus, detection bias may exist.

\section{Conclusions}

In this study, we have shown the translational value of radiomics in assisting with the timing of management of nodules detected with low-dose CT in lung cancer screening. Considering the lack of an established evidence-based protocol for establishing such schedules, further validation is required to optimize the time targets in lung cancer screening.

\section{Abbreviations}

AATS: American association for thoracic surgery; ACCP: American college of chest physicians; AUCt: Area under curve by time-dependent definition; $\mathrm{Cl}$ : Confidence interval; CT: Computed tomography; LongHEM: Long-run high gray-level emphasis mean; Lung-RADS: Lung CT Screening Reporting and Data System; NCCN: National comprehensive cancer network; ROI: Region-ofinterest; RSF: Random survival forest.

\section{Supplementary Information}

The online version contains supplementary material available at https://doi. org/10.1186/s12967-021-02849-8.

Additional file 1: Method S1. Region-of-interest delineation. Method S2. Radiomic feature definition and calculation. Method S3. Radiomic feature selection. Method S4. Biomarker development. Table S1. Summary of selected radiomic features. Table S2. Cross validation of the radiomics model. Figure S1. Variable importance of the selected radiomic features

\section{Acknowledgements}

Not applicable.

\section{Authors' contributions}

JJ and WS conceptualized this study. FZ, XS and XX did the data collection, curation and interpretation. ZW, NL, WH, FX, CY, YH and LW performed the formal analysis. ZW wrote the original draft, and all the authors substantively revised it. All authors read and approved the final manuscript.

\section{Funding}

This work was supported by the CAMS Innovation Fund for Medical Sciences [Grant Number: 2017-12M-1-009], the PUMC Youth Fund and the Fundamental Research Funds for the Central Universities [Grant Number: 2017310049], and the PUMC Innovation Fund for Graduate Student [Grant Number: 2018-100201-21]. The funders have no roles in the design of the study and collection, analysis, interpretation of data or in writing the manuscript.

\section{Availability of data and materials}

The datasets used during the current study are available from the corresponding author on reasonable request.

\section{Declarations}

\section{Ethics approval and consent to participate}

The study protocol was approved by the Ethics Review Board of the Institute of Basic Medical Sciences, Chinese Academy of Medical Sciences. The necessity for written informed consent was waived, as the data were analyzed anonymously.

Consent for publication

Not applicable. 


\section{Competing interests}

The authors declare that they have no competing interests.

\section{Author details}

${ }^{1}$ Department of Epidemiology and Biostatistics, Institute of Basic Medical Sciences, Chinese Academy of Medical Sciences/School of Basic Medicine, Peking Union Medical College, Beijing, China. ${ }^{2}$ Department of Radiology, Peking Union Medical College Hospital, Chinese Academy of Medical Sciences, Beijing, China. ${ }^{3}$ Department of Radiology, Beijing Chao-Yang Hospital, Capital Medical University, Beijing, China.

Received: 14 January 2021 Accepted: 18 April 2021

Published online: 04 May 2021

\section{References}

1. Bi WL, Hosny A, Schabath MB, Giger M, Birkbak NJ, Mehrtash A, et al. Artificial intelligence in cancer imaging: clinical challenges and applications. CA Cancer J Clin. 2019:69:127-57.

2. de Koning $H J$, van der Aalst CM, de Jong PA, Scholten ET, Nackaerts K, Heuvelmans MA, et al. Reduced lung-cancer mortality with volume CT screening in a randomized trial. N Engl J Med. 2020;382:503-13.

3. National Lung Screening Trial Research Team. Reduced lung-cancer mortality with low-dose computed tomographic screening. N Engl J Med. 2011;365:395-409.

4. Pastorino U, Silva M, Sestini S, Sabia F, Boeri M, Cantarutti A, et al. Prolonged lung cancer screening reduced 10-year mortality in the MILD trial: new confirmation of lung cancer screening efficacy. Ann Oncol. 2019;30:1162-9.

5. Mcwilliams A, Tammemagi MC, Mayo JR, Roberts H, Liu G, Soghrati K, et al. Probability of cancer in pulmonary nodules detected on first screening CT. N Engl J Med. 2013;369:910-9.

6. NCCN 2020. NCCN Clinical Practice Guidelines in Oncology. Lung Cancer Screening. Version 1. 2020. https://www.nccn.org/professionals/physi cian_gls/pdf/lung_screening.pdf. Accessed 14 Jan 2021.

7. Zhou Q, Fan Y, Wang Y, Qiao Y, Wang G, Huang Y, et al. China national lung cancer screening guideline with low-dose computed tomography (2018 Version). Zhongguo Fei Ai Za Zhi. 2018;21:67-75.

8. Macmahon H, Bankier AA, Naidich DP. Lung cancer screening: what is the effect of using a larger nodule threshold size to determine who is assigned to short-term CT follow-up? Radiology. 2014;273:326-7.

9. van Riel S, Jacobs C, Scholten ET, Wittenberg R, Wille MMW, de Hoop $B$, et al. Observer variability for lung-RADS categorisation of lung cancer screening CTs: impact on patient management. Eur Radiol. 2019:29:924-31.

10. van Riel SJ, Sánchez Cl, Bankier AA, Naidich DP, Verschakelen J, Scholten ET, et al. Observer variability for classification of pulmonary nodules on low-dose CT images and its effect on nodule management. Radiology. 2015;277:863-71.

11. Doubeni CA, Gabler NB, Wheeler CM, McCarthy AM, Castle PE, Halm EA, et al. Timely follow-up of positive cancer screening results: a systematic review and recommendations from the PROSPR Consortium. CA Cancer J Clin. 2019;68:199-216.

12. Aerts HJ, Velazquez ER, Leijenaar RT, Parmar C, Grossmann P, Bussink SCJ, et al. Decoding tumour phenotype by noninvasive imaging using a quantitative radiomics approachapproach. Nat Commun. 2014:5:4006.

13. Traverso A, Wee L, Dekker A, Gillies R. Repeatability and reproducibility of radiomic features: a systematic review. Int J Radiat Oncol Biol Phys. 2018;102:1143-58.

14. Ishwaran H, Kogalur UB, Chen X, Minn AJ. Random survival forests for high-dimensional data. Stat Anal Data Min. 2011;4:115-32.
15. American College of Radiology. Lung CT Screening Reporting \& Data System (Lung-RADS). https://www.acr.org/Clinical-Resources/Reportingand-Data-Systems/Lung-Rads. Accessed Jan 14, 2021.

16. Gould MK, Donington J, Lynch WR, Mazzone PJ, Midthun DE, Naidich DP, et al. Evaluation of individuals with pulmonary nodules: when is it lung cancer? diagnosis and management of lung cancer, 3rd Ed: American college of chest physicians evidence-based clinical practice guidelines. Chest. 2013;143:e93S-120S.

17. Jaklitsch MT, Jacobson FL, Austinet JH, Field JK, Jett JR, Keshavjee S, et al. The American Association for Thoracic Surgery guidelines for lung cancer screening using low-dose computed tomography scans for lung cancer survivors and other high-risk groups. J Thorac Cardiovasc Surg. 2012;144:33-8

18. Heagerty PJ, Zheng Y. Survival model predictive accuracy and ROC curves. Biometrics. 2005;61:92-105.

19. Choi W, Oh JH, Riyahi S, Liu CJ, Jiang F, Chen W, et al. Radiomics analysis of pulmonary nodules in low-dose CT for early detection of lung cancer. Med Phys. 2018:45:1537-49.

20. Sun Q, Huang Y, Wang J, Zhao S, Zhang L, Tang W, et al. Applying CT texture analysis to determine the prognostic value of subsolid nodules detected during low-dose CT screening. Clin Radiol. 2019;74:59-66.

21. Hawkins S, Wang H, Liu Y, Garcia A, Stringfield O, Krewer H, et al. Predicting malignant nodules from screening CT scans. J Thorac Oncol. 2016:11:2120-8.

22. Zwanenburg A, Leger S, Agolli L, Pilz K, Troost EGC, Richter C, et al. Assessing robustness of radiomic features by image perturbation. Sci Rep. 2019;9:614.

23. Lu L, Ehmke RC, Schwartz LH, Zhao B. Assessing agreement between radiomic features computed for multiple $C T$ imaging settings. PLOS ONE. 2016;11:e0166550.

24. Mao L, Chen H, Liang M, Li K, Gao J, Qin P, et al. Quantitative radiomic model for predicting malignancy of small solid pulmonary nodules detected by low-dose CT screening. Quant Imaging Med Surg. 2019;9:263-72.

25. Winter A, Aberle DR, Hsu W. External validation and recalibration of the brock model to predict probability of cancer in pulmonary nodules using NLST data. Thorax. 2019;74:551-63.

26. Kamiya A, Murayama S, Kamiya H, Yamashiro T, Oshiro Y, Tanaka N. Kurtosis and skewness assessments of solid lung nodule density histograms: differentiating malignant from benign nodules on CT. Jpn J Radiol. 2014;32:14-21.

27. Aerts HJ, Velazquez ER, Leijenaar RT, Parmar C, Grossmann P, Carvalho $S$, et al. Decoding tumour phenotype by noninvasive imaging using a quantitative radiomics approachapproach. Nat Commun. 2014;5:4006.

28. McKee BJ, Regis SM, McKee AB, Flacke S, Wald C. Performance of ACR lung-RADS in a clinical CT lung screening program. J Am Coll Radiol. 2016;13:R25-9.

29. Li Q, Balagurunathan Y, Liu Y, Qi J, Schabath MB, Ye Z, et al. Comparison between radiological semantic features and Lung-RADS in predicting malignancy of screen-detected lung nodules in the national lung screening trial. Clin Lung Cancer. 2018;19(148-56):e3.

30. Sun R, Limkin EJ, Vakalopoulou M, Dercle L, Champiat S, Hanet SR, et al. A radiomics approach to assess tumour-infiltrating CD8 cells and response to anti-PD-1 or anti-PD-L1 immunotherapy: an imaging biomarker, Retrospective Multicohort Study. Lancet Oncol. 2018;19:1180-91.

\section{Publisher's Note}

Springer Nature remains neutral with regard to jurisdictional claims in published maps and institutional affiliations. 